\title{
SILVER RELEASED FROM WOUND DRESSINGS DAMAGE SKIN FIBROBLASTS AND KERATINOCYTES AND EX VIVO SKIN
}

\author{
Pavlík V.',2, Nešporová, K.', Vágnerová, H.', Šafránková B,' Brůnova Z.', Velebný, V.' \\ Contipro a.s., Dolní Dobrouč 401, Dolní Dobrouč, Czech Republic

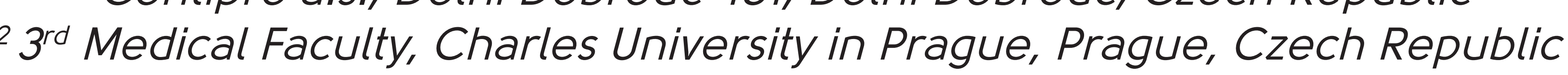

\section{Introduction}

Silver is a favourite antiseptic used in wound healing products, that acts through generation of reactive oxygen species. Yet the oxidative damage aimed at bacteria may as well affect patient's cells. Silver toxicity was observed in vitro on human normal skin and diabetic wound fibroblasts (Poon \& Burd, 2004; Zou et al., 2013). In addition, silver sulfadiazine retarded wound healing in mice (Rosen et al., 2015) and in human (Fumal et al., 2002).

There are various forms of silver (silver salt, nanocrystalline, nanoparticles) and the results from in vitro experiments are not easily translatable to a whole skin. Therefore, we evaluated in vitro and ex vivo toxicity of three bandages with silver that are used in clinical praxis

\section{Results}

We investigated properties of a bandage with silver nanoparticles and carboxymethyl cellulose (Ag-CMC), silver nanoparticles, CMC and alginate (Ag-CMC-ALG) and nanocrystalline silver on polyester (Ag0-PE). We observed significant cytotoxicity of the eluates from the three bandages on normal dermal fibroblasts (NHDF) and keratinocytes (NHEK) (Figure 1A and 1B). The significant cytotoxic effect was apparent after 24 hours, when there were less than $30 \%$ of viable cells compared to the control in MTT test. The negative effect on the skin cells was further documented with fluorescence microscopy, which illustrate altered morphology and decreased viability of both NHDF and NHEK (Figure IC). Interestingly, Ag-CMC-ALG bandage had the least damaging effect. Contact cytotoxicity of the bandages was assessed on monolayers of 3T3 fibroblasts for 24 hours (Figure ID), clear halo zones around the bandages indicate dissolution of silver and affecting surrounding cells. We conclude that fibroblasts exposed to the silver bandage eluates die in necrosis rather than apoptosis, as indicated by flow cytometry staining with propidium iodide and annexin V (Figure IE). The silver may affect not only fibroblasts and keratinocytes, immune cells from full human blood were stimulated by eluates from silver bandages in MAT test for 24 hours and secreted TNF- $a$ and IL-6, as seen in Figure IF.
A

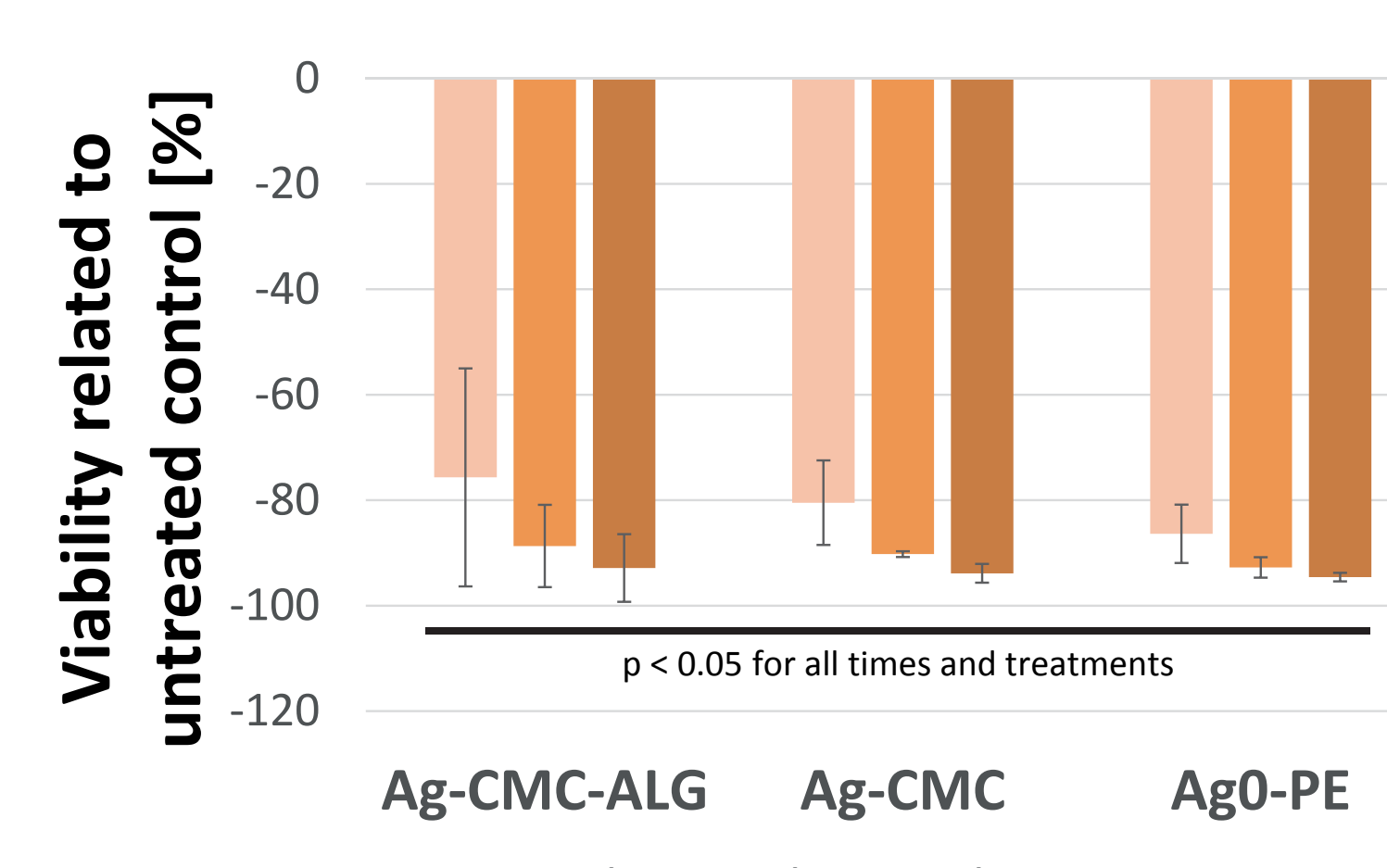

C

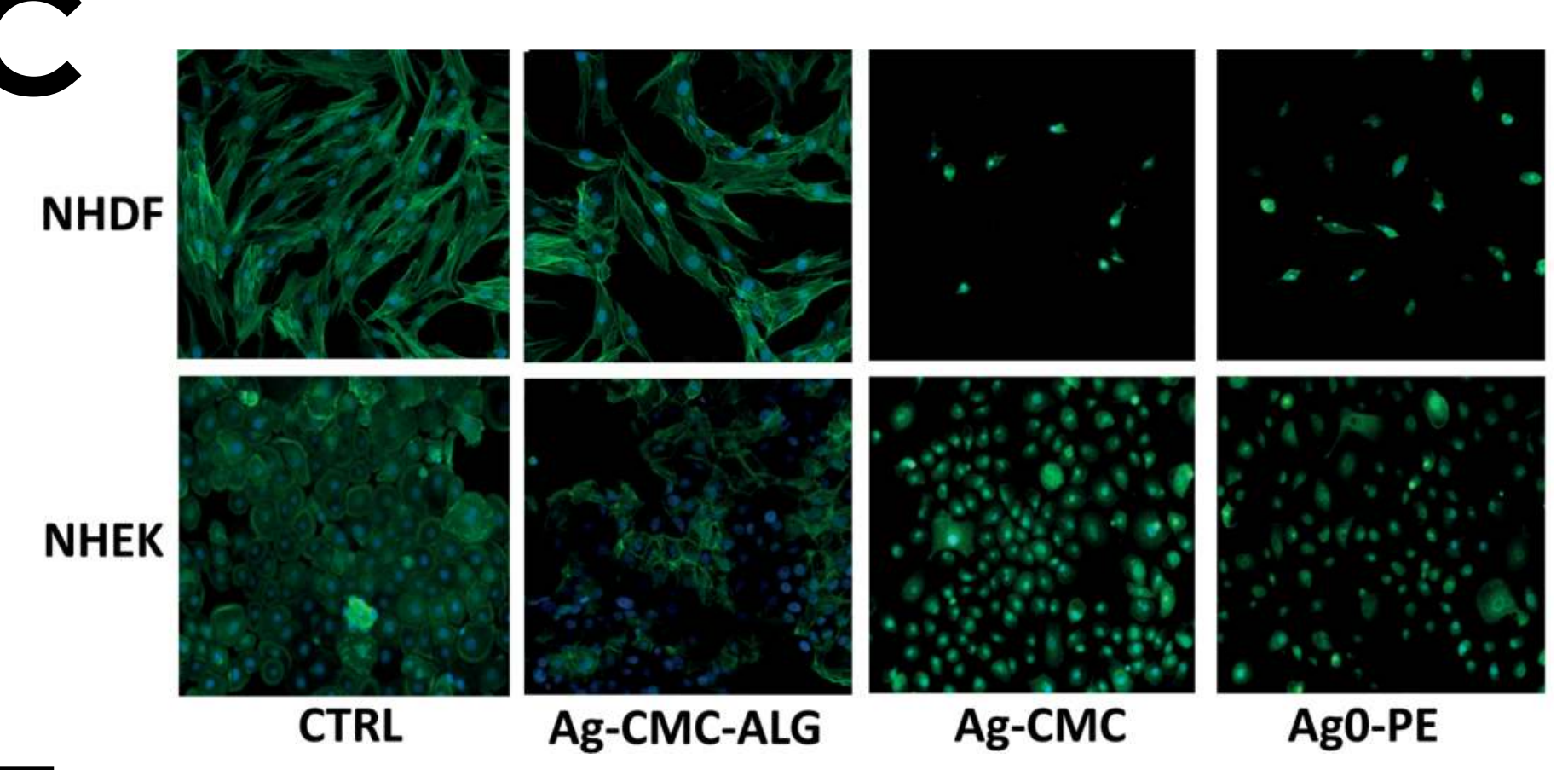

$\mathbf{E}$

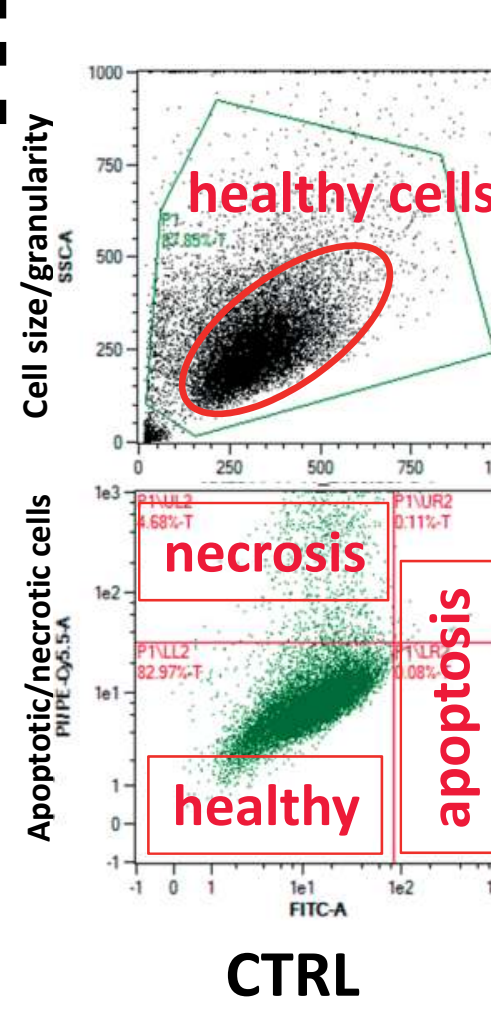

B

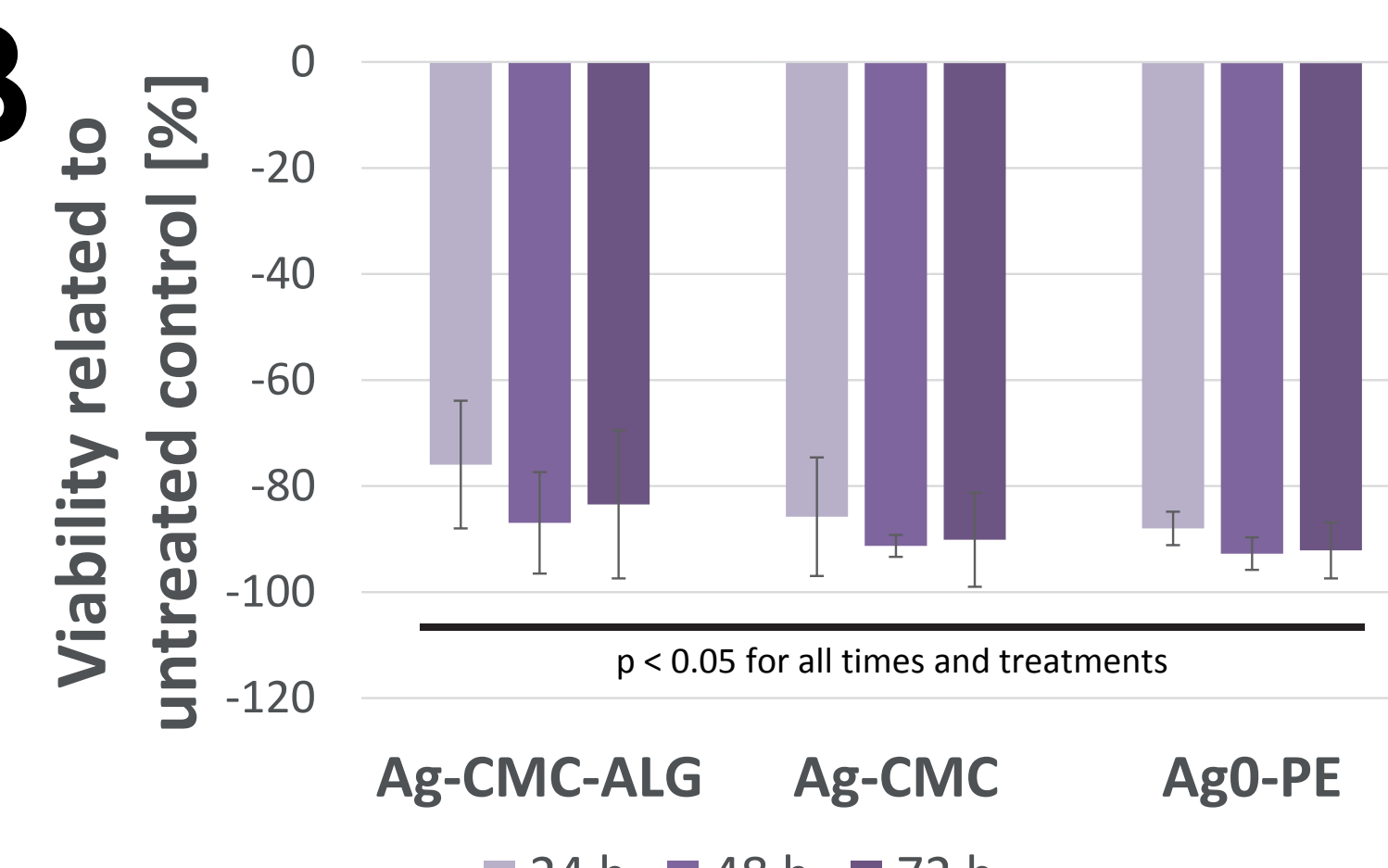

D $24 \mathrm{~h} \square 48 \mathrm{~h}-72 \mathrm{~h}$<smiles>[Mg][Mg]</smiles>

$\mathbf{F}$

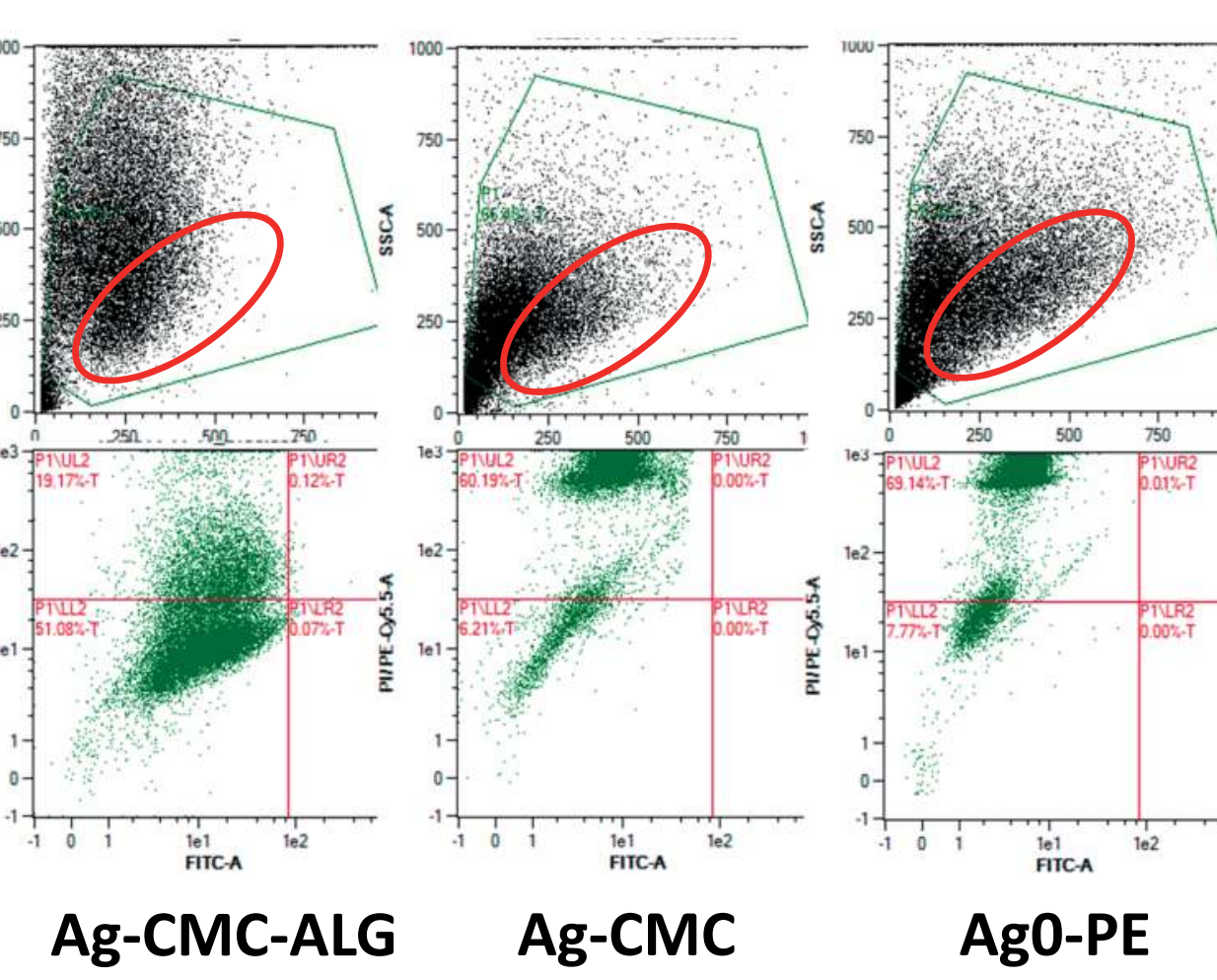

A

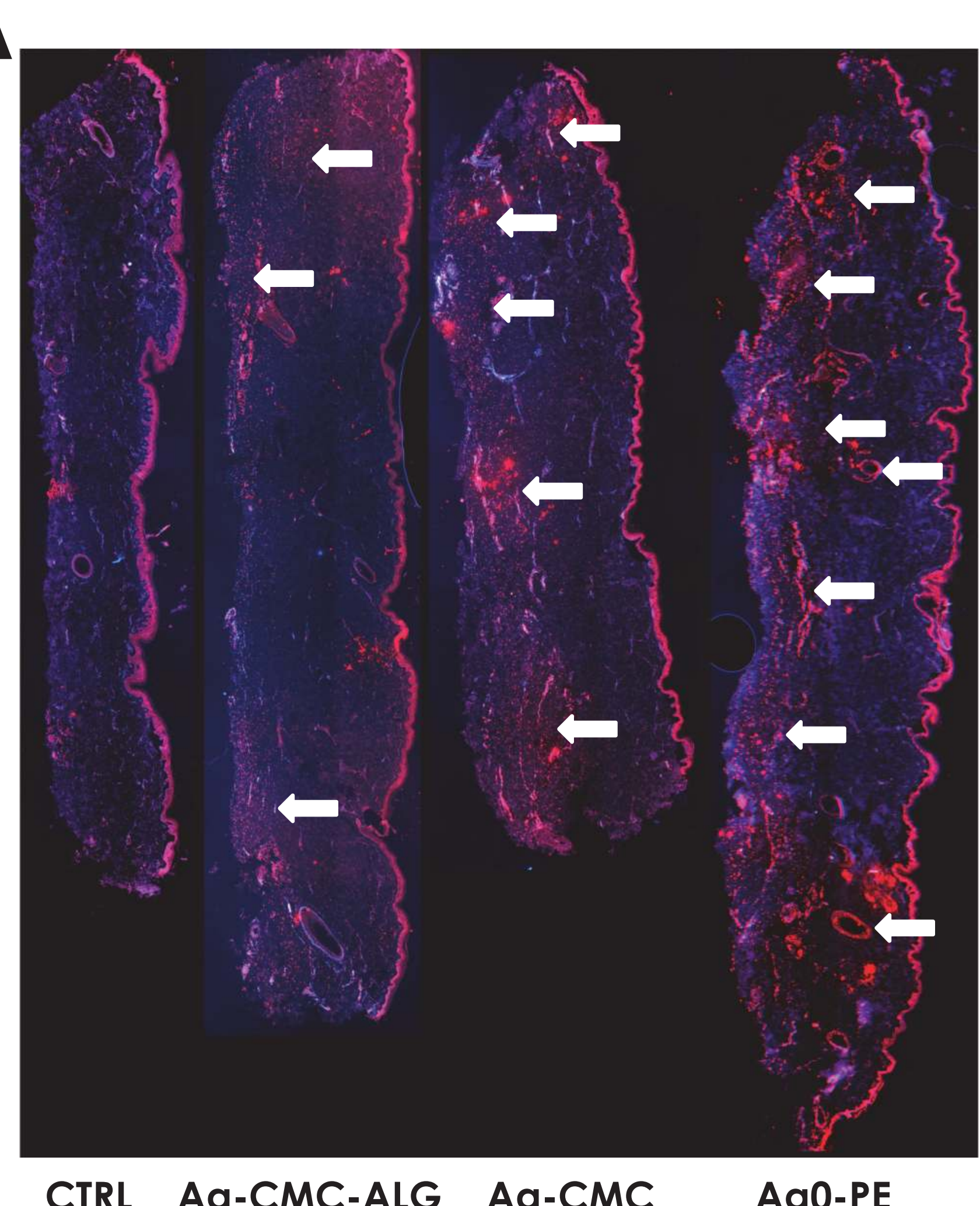

C

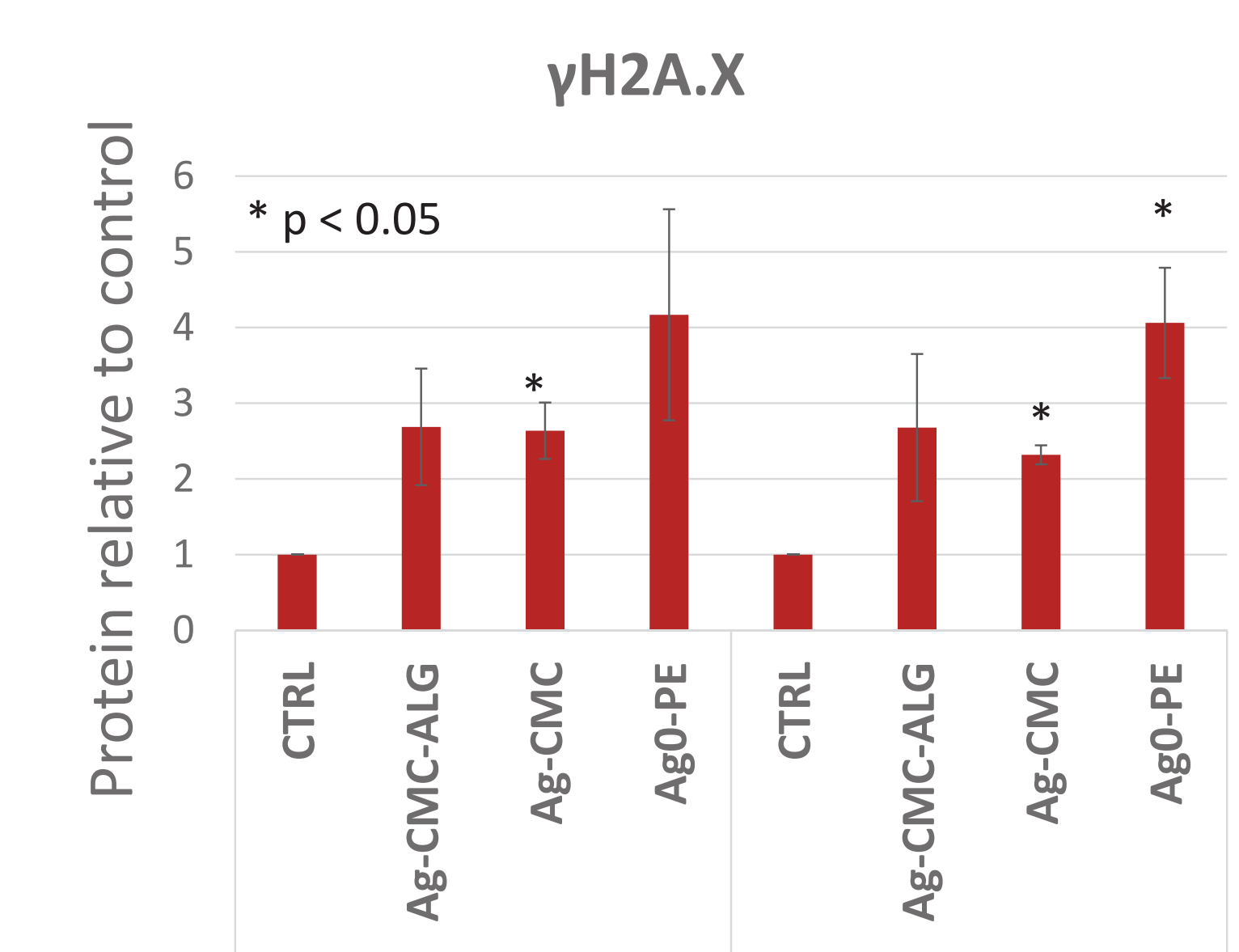

B

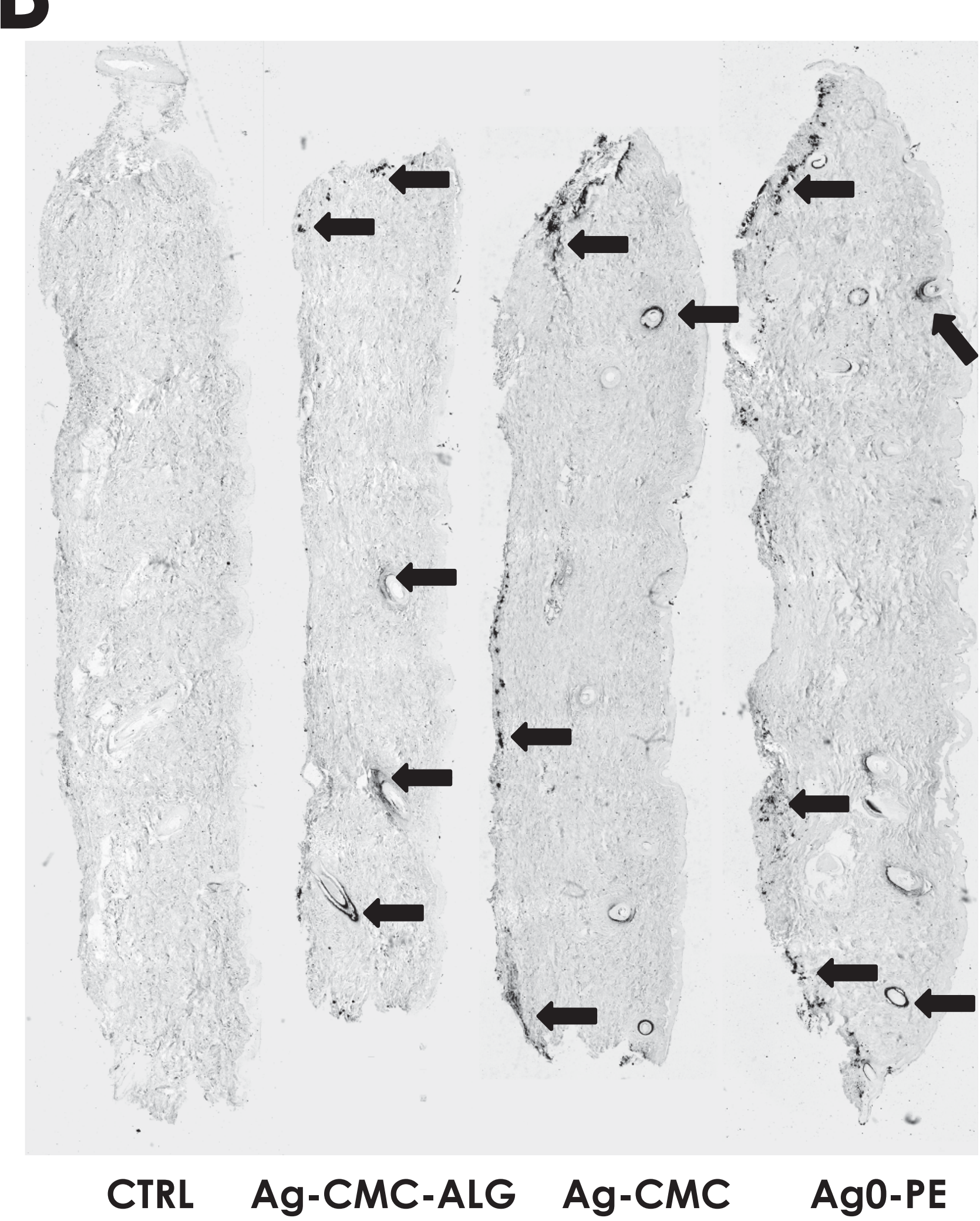

D

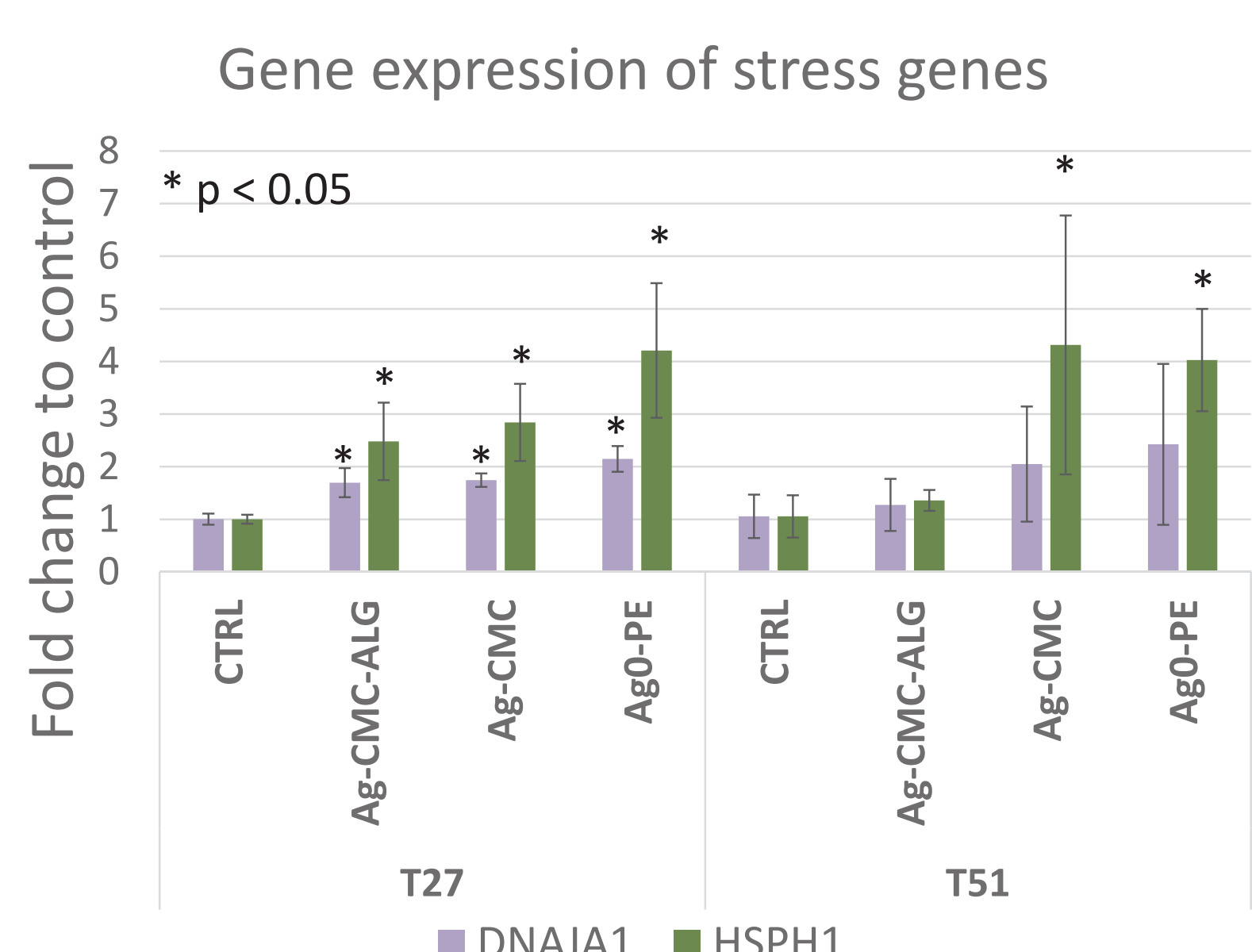

Figure 2. (A) Collection of histological sections of ex vivo porcine skin treated for $51 \mathrm{~h}$ with silver bandages placed on the dermal side (left on each section) and subsequently stained for $\gamma H 2 A$ .$X$ (red dots, areas marked with arrows). (B) Silver was detected in the corresponding sections with autometallography (arrows). (C) YH2A.X quantification with WB after cultivation for 27 or $5 \mathrm{l} h$ with the bandages; mean \pm SEM, $n=4 ;$; $t$-test to CTRL.. (D) Gene expression of stress-related $51 \mathrm{~h}$ with the bandages; mean $\pm S E M, n=4$; $t$-test to $C T R L$. (D) Gene expression of stress-rel
genes after 27 or $51 \mathrm{~h}$ of incubation with the bandages; mean $\pm S D n=4$, test to CTRL.

We investigated, if the silver toxicity manifest itself as well in the context of full profile porcine skin. The excised skin from porcine auricles was placed dermal side upwards into DMEM cultivation media and the moistened bandages or a cotton gauze (CTRL) covered the dermis. We observed strong staining of a prototypic DNA double-strand break marker $\mathrm{\gamma H} 2 \mathrm{~A}$.X inside the tissue (Figure 2A). We also detected silver with autometallography In the corresponding sections (Figure 2B). The strong silver build-up and $\mathrm{\gamma H} 2 \mathrm{~A}$.X signal was apparent not only in the stromal cells of the dermis, but also in the endothelia. Quantification of the $\mathrm{\gamma H} 2 \mathrm{~A} . \mathrm{X}$ in the tissues by Western blotting showed increase of $\gamma \mathrm{H} 2 \mathrm{~A}$. $X$ for all the silver bandages, however not statistically significant for the Ag-CMC-ALG bandage (Figure 2C). All of the silver bandages increased significantly gene expression of stress-related genes HSPHI (heat shock protein family $\mathrm{H} \mathrm{l}$ ) and DNAJAl (DnaJ heat shock protein family member Al) after $27 \mathrm{~h}$ of incubation (Figure 2D).

\section{Conclusion}

We demonstrated that the eluates from silver bandages induced necrosis of skin cells in vitro. In addition, silver penetrated into the porcine skin and induced there DNA damage and stress response. These mechanisms are in line with the proclaimed antiseptic activity on the bacterial cells, however due to nonspecificity of the mechanism, they can inflict a collateral damage on skin cells. Therefore, prophylactic use of silver bandages may unnecessarily burden the wound cells. Less penetrating or biocompatible (not a heavy metal) antiseptics may be less harmful to cells during wound healing.
Figure 1. Toxicity of silver bandages to NHDF (A) and NHEK (B) assessed with MTT test; mean $\pm S D$, $n=4, t$-test to CTRL. (C) Actin (green) and nuclear (blue) staining of NHDF or NHEK treated for $24 \mathrm{~h}$ with bandage eluates. (D) Contact toxicity of the bandages to $3 T 3$ fibroblasts and crystal violet staining. Squares indicate placement of the bandages. (E) FCM analysis of apoptosis or necrosis of NHDF treated for $24 \mathrm{~h}$ with the bandage eluates. (F) TNF-a and IL-6 secretion by blood cells in MAT test. 